\title{
Simplified Probabilistic Analysis of Settlement of Cyclically Loaded Soil Stratum by Point Estimate Method
}

\author{
Jarosław Przewłócki*, Jarosław Górski**, Waldemar Świdziński*** \\ ${ }^{*}$ Prof. D. Sc., Faculty of Architecture, Gdańsk University of Technology, Narutowicza 11/12, 80-233 \\ Gdańsk, Poland,jprzew@pg.gda.pl, \\ ${ }^{* *} \mathrm{Ph}$. D, D. Sc., Faculty of Civil and Environmental Engineering, Gdańsk University of Technology, \\ Narutowicza 11/12, 80-233 Gdańsk, Poland, jgorski@pg.gda.pl, \\ ${ }^{* * *}$ Ph. D, D. Sc., Institute of Hydro-Engineering, Polish Academy of Sciences, Kościerska 7, 80-328 \\ Gdańsk, Poland, waldek@ibwpan.gda.pl
}

(Received July 21, 2016; revised January 05, 2017)

\begin{abstract}
The paper deals with the probabilistic analysis of the settlement of a non-cohesive soil layer subjected to cyclic loading. Originally, the settlement assessment is based on a deterministic compaction model, which requires integration of a set of differential equations. However, with the use of the Bessel functions, the settlement of a soil stratum can be calculated by a simplified algorithm. The compaction model parameters were determined for soil samples taken from subsoil near the Izmit Bay, Turkey. The computations were performed for various sets of random variables. The point estimate method was applied, and the results were verified by the Monte Carlo method. The outcome leads to a conclusion that can be useful in the prediction of soil settlement under seismic loading.
\end{abstract}

Key words: soil settlement, seismic loading, random material parameters, point estimate method

\section{Introduction}

There are several studies on non-cohesive soil subjected to cyclic loading under drained conditions. Most of them concern experimental results. Some focus attention on empirical or semi-empirical models, but only a few analyze the problem theoretically. It seems that the theory of compaction proposed by Sawicki and Morland (1986) is one of the most advanced. It reproduces the response of cyclically loaded soil quite accurately. A modified engineering version of this theory, the so-called compaction model for amplitudes, was proposed by Sawicki (1987). A good agreement between the calculated results and the measured values proves the usefulness of this model in estimating the settlement of cyclically loaded non-cohesive soils.

In order to develop a rational framework including material, load and model uncertainties, a probabilistic analysis should be introduced. The serviceability limit state 
in the probabilistic approach is usually related to settlements of foundations. Brząkała and Puła (1996) analyzed the settlement of shallow foundations resting on a layered subsoil, using the finite element method (FEM) coupled with probabilistic versions of the perturbation and the Neumann expansion methods. Gordon, Fenton and Griffiths (2002) considered soils as spatially random media and analyzed settlement under spread footings by the FEM combined with the Monte Carlo method (MCM). Bauer and Puła (2000) applied the response surface and second-order reliability methods (SORM) to estimate the reliability index associated with exceeding a certain allowable settlement of a shallow foundation. The settlement of loose granular materials subjected to surface loads can also be investigated from the viewpoint of probabilistic mechanics of particular media (Bourdeau and Harr 1989). Strip foundations for both 2-D and 3-D states of stresses and strains were analyzed by a probabilistic FEM in (Przewłócki 1999, Przewłócki and Górski 1999). No publication has been found concerning the random approach to the problem of the settlement of subsoil without an embankment or foundation loading. However, these cases may be significant, too.

The point estimate method (PEM), proposed by Rosenblueth (1975), has been widely adopted in geotechnical reliability analyses. Several researchers, including Harr (1989), Lind (1983), Li (1992) and Hong (1998), have modified the Rosenblueth procedure to optimize computational accuracy and effort. The case of a large number of variables is widely discussed by Christian and Baecher (1999). Suchomel and Mašín (2011) used various probabilistic methods to analyze a strip footing on a horizontally stratified sandy deposit. They found the basic PEM to be the most accurate method. Sayed et al (2008) carried out a reliability analysis, using different probabilistic methods to study the stability aspects of reinforced soil retaining walls under static and seismic conditions. Baecher and Christian (2003), and Przewłócki (2006) used the PEM to assess the load-bearing capacity of a footing. Fattah (2010) used this method to estimate the bearing capacity of axially loaded piles. Gibson (2011), Wang and Huang (2012) applied the PEM for the design of slopes.

This paper presents a use of the PEM for the analysis of the settlement of a soil layer subjected to cyclic loading. Soil parameters, including compaction model constants and cyclic load parameters, were assumed random. The parameters were determined for soil samples taken from subsoil near the Izmit Bay, Turkey. This region was hit by a severe earthquake in 1999, causing both soil liquefaction and significant subsoil settlements (Sawicki and Świdziński 2006). The mean value and the standard deviation of the settlement were estimated. The results were verified by means of the MCM. Although the problem regards the soil layer only, it is also important for any soil-structure interaction issues, e.g. foundation settlement.

\section{Compaction Model}

Based on the results of cyclic simple shear tests for non-cohesive soils, the following constitutive equation was proposed by Sawicki (1987): 


$$
\frac{d \Phi}{d N}=C_{1} J \exp \left(-C_{2} \Phi\right)
$$

where $\Phi=\left(n_{0}-n\right) / n_{0}$ or $\Phi=\varepsilon_{v}\left(1-n_{0}\right) / n_{0}$ is a relative change in porosity corresponding to irreversible volumetric changes $\varepsilon_{v}$ (compaction), $n$ and $n_{0}$ are the actual and initial porosities, $C_{1}$, and $C_{2}$ are material constants determined for a given soil from cyclic simple shear tests, $J$ is the second invariant of the deviator of cyclic strain amplitudes, and $N$ is the number of loading cycles (Sawicki and Świdziński 2006, 2007). Equation (1) is valid for a general type of non-cohesive soil and history of cyclic loading. In order to compute the densification $\Phi$, the distribution of strain amplitudes in the soil mass should be known.

The second constitutive equation describes the relation between the deviators of cyclic stress $\boldsymbol{S}$ and strain $\boldsymbol{Y}$ amplitude tensors:

$$
\boldsymbol{S}=G \boldsymbol{Y},
$$

where $G$ is a generalized shear modulus, which depends mainly on the mean effective stress and the amplitude of shear strains. For strains smaller than $10^{-4}$, a sufficiently good approximation for $G$ may be given in the form proposed by Martin et al (1975):

$$
G=G_{0} \sqrt{\frac{p^{\prime}}{p_{0}}}
$$

where $G_{0}$ is a soil constant, $p^{\prime}$ is the mean effective stress, and $p_{0}$ is the stress unit equal to $10^{5} \mathrm{~N} / \mathrm{m}^{2}$.

The mean effective stress for the homogeneous layer considered in this paper takes the form:

$$
p^{\prime}=\frac{1}{3\left(1+2 K_{0}\right) \gamma H(1-Z)},
$$

where $\gamma$ is the unit weight of the soil, $K_{0}$ is the coefficient of earth pressure at rest, and the parameters $H$ and $Z$ are shown in Fig. 3.

The relationships (1-3) lead to the description of volumetric changes in dry non-cohesive soil (or saturated soil under free drainage conditions) caused by cyclic shearing for a given loading history. The simplicity of the proposed model is due to the fact that there are only four model parameters, $C_{1}, C_{2}, G_{0}$ and $n_{0}$ to be determined experimentally in the laboratory.

\section{Specification of Soil Properties}

The settlement analysis was carried out for a $10 \mathrm{~m}$ thick sandy layer of subsoil near the seacoast of the Izmit Bay, Turkey (Sawicki and Świdziński 2006). The grain size distribution of that soil classifies it as gravelly sand of a density $\rho=1720 \mathrm{~kg} / \mathrm{m}^{3}$. The values of compaction coefficients in Eq. 1 were determined from cyclic simple shear 
test results. The tests were performed on reconstituted specimens of dry soil deposited by the air pluviation method in an originally designed and manufactured apparatus (Sawicki and Świdziński 2006). The reconstituted specimens were next subjected to cyclic loading with various shear strain amplitudes $\gamma_{0}$ and constant mean stress (in the case of dry soil, effective stress is equivalent to total stress, $p^{\prime}=p$ ). In total, four series corresponding to four different cyclic shear strain amplitudes were carried out. In each experiment, the settlement of the sample caused by cyclic loading versus the number of loading cycles $N$ was recorded.

The basic hypothesis of a compaction model assumes that, for a given non-cohesive soil subjected to cyclic loading, there exists a unique common compaction curve describing its capacity to densify (Sawicki 1987). According to this hypothesis, various compaction curves obtained for various shear strain amplitudes can be represented by the so-called common compaction curve with a new representation of the results. In this approach, the number of loading cycles $N$ is replaced by a new model variable $\xi=J N$. Reinterpreted results of 14 tests of cyclic simple shear at different shear strain amplitudes are presented in Fig. 1.

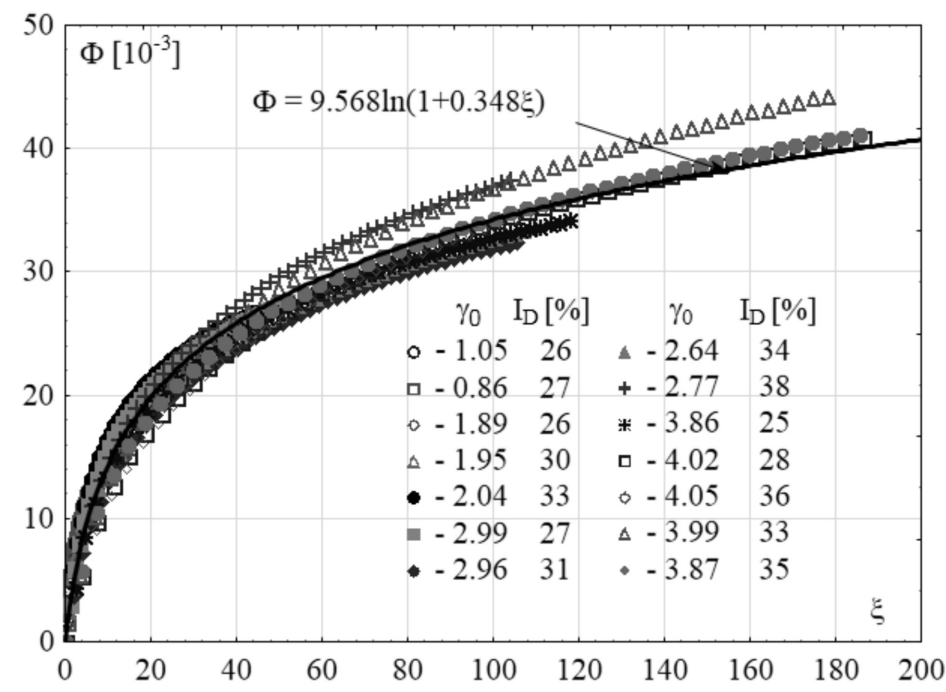

Fig. 1. Common compaction curve for the soil tested

In the case of simple cyclic shear with a constant strain amplitude $\gamma_{0}$, the second invariant of the deviator of the cyclic strain amplitude $J$ takes a simple form:

$$
J=\frac{1}{4} \gamma_{0}^{2}
$$

Assuming that during a given load step the material behaves elastically and its parameters are constant while the number of cycles is a continuous variable, a common compaction curve can be approximated by the following relation: 


$$
\Phi=D_{1} \ln \left(1+D_{2} \xi\right),
$$

where $D_{1}$ and $D_{2}$ are coefficients that can be determined by the least square method. Such a common compaction curve for the soil tested is shown in Fig. 1.

The relationships between the parameters $D_{1}, D_{2}$ and the parameters $C_{1}, C_{2}$ in Eq. (1) are given by the following formulae (Sawicki 1987):

$$
C_{1}=\frac{1}{D_{2}}, \quad C_{2}=D_{1} D_{2}
$$

If the strain amplitude applied at the boundary is constant, expression (6) stands for compaction caused by a specified number of cycles. If the boundary strain amplitudes vary, the compaction is calculated separately for each section of loading history with a constant amplitude and is accumulated.

The impact of the shear modulus on the mean effective stress given by Eq. (3) was determined from the results of cyclic triaxial compression tests. In a single experiment, the specimen was first anisotropically pre-consolidated to the assumed stress deviator and the mean effective stress, and then cyclically subjected to shear stress of a given amplitude while the cell pressure was kept constant. The shear modulus $G$ was determined from the loading-unloading-reloading hysteresis loop as a secant of a given shear stress amplitude. In order to find the relationship given by Eq. 3, the tests were repeated at different levels of the mean effective stress (Sawicki and Świdziński 2006). The mean values of the shear modulus for the mean effective stress applied are shown in Fig. 2.

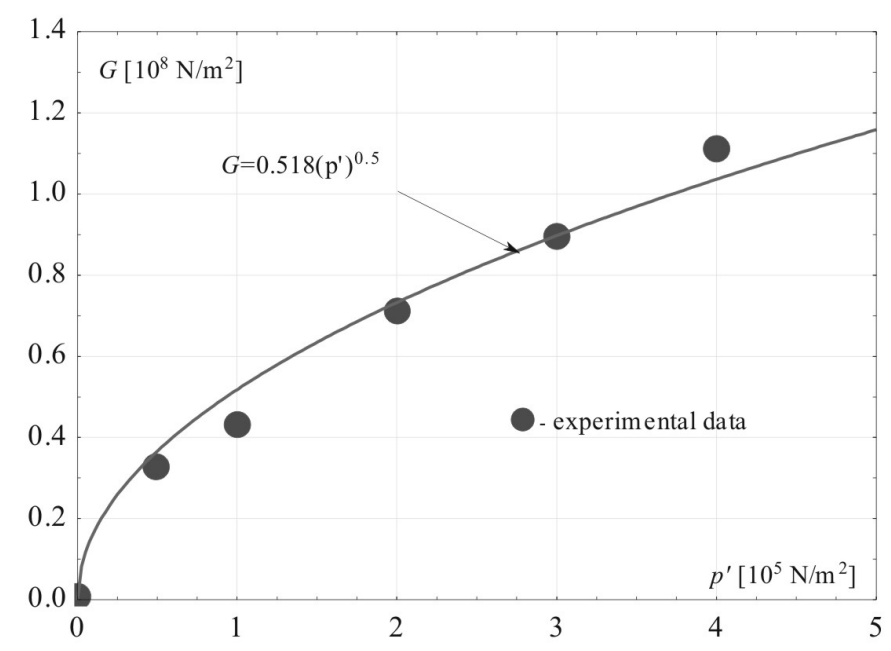

Fig. 2. Shear modulus as a function of the mean effective stress determined by cyclic triaxial compression tests 
Approximation of the test results presented in Fig. 2 by relationship (3) made it possible to determine the coefficient $G_{0}=0.518$. The coefficient is expressed by the same unit as the $G$ modulus, i.e. $10^{8} \mathrm{~N} / \mathrm{m}^{2}$. It should be pointed out that the value is very close to the commonly accepted magnitude in geotechnics.

The parameters of compaction curves $D_{1}$ and $D_{2}$ (Eq. 6) are approximated values of laboratory test results. Thus, they are random parameters. Regression analysis performed by means of the Statistica package with the data presented in Fig. 1 resulted in the following estimators of mean values $m_{D}$ and standard deviation: $m_{D 1}=9.568$, $\sigma_{D_{1}}=1.164, m_{D_{2}}=0.348, \sigma_{D_{2}}=0.129$. Correlation between the parameters was also assessed: $r_{D_{1} D_{2}}=-0.968$. A similar procedure was applied to the statistical parameters of the coefficient $G_{0}: m_{G_{0}}=0.518 \times 10^{8} \mathrm{~N} / \mathrm{m}^{2}, \sigma_{G_{0}}=0.036 \times 10^{8} \mathrm{~N} / \mathrm{m}^{2}$. Next, the mean value and standard deviation of porosity were obtained directly from laboratory tests: $m_{n_{0}}=0.409, \sigma_{n_{0}}=0.01$. The small value of the standard deviation of porosity results from the fact that all specimens in the cycle simple shear test were reconstituted to have similar density (medium dense sand), since the model proposed does not take into account the initial state of the non-cohesive soil. The same regards the small value of standard deviation for the coefficient $G_{0}$.

The influence of correlation between compaction parameters should also be investigated. For physical reasons, the correlation between $n_{0}$ and $G_{0}$ is negative, whereas the correlation between $n_{0}$ and $D_{1}$ is positive. Unfortunately, the available experimental data are insufficient for a thorough quantitative analysis. Thus, the following coefficients were proposed: $r_{D_{1} G_{0}}=-0.5, r_{D_{1} n_{0}}=0.5, r_{n_{0} G_{0}}=-0.5, r_{D_{2} G_{0}}=0.5$, and $r_{D_{2} n_{0}}=-0.5$.

\section{Boundary Problem - Deterministic Solution}

A non-cohesive elastic soil layer of thickness $H$ and specific density $\rho_{0}\left(\mathrm{~kg} / \mathrm{m}^{3}\right)$ resting on a rough rigid base (Fig. 3 ) is considered.

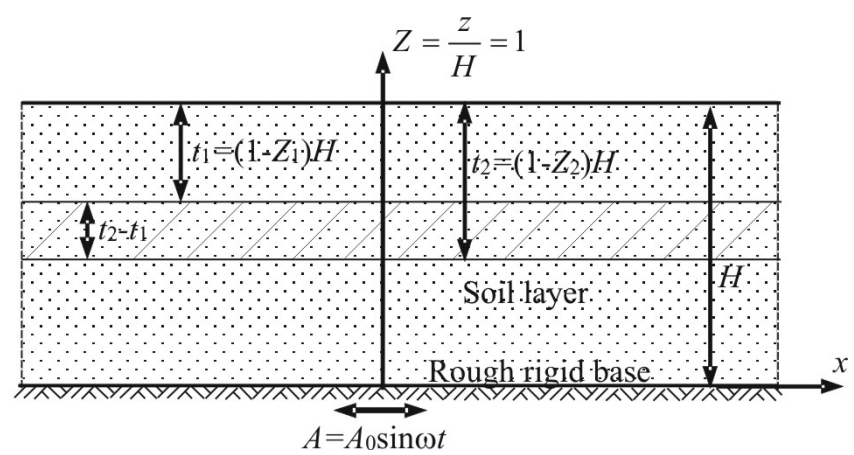

Fig. 3. Initial coordinate system and the coordinate system for the analysis

The soil stratum is subjected to a horizontal acceleration $A=A_{0} \sin \omega t$ applied at the rigid base in order to reflect seismic loading due to an earthquake. Here $A_{0}$ 
is the maximum amplitude of the horizontal sinusoidal acceleration at the ground surface, and $\omega$ is the frequency. It is reasonable to analyze a one-dimensional problem corresponding to a two-dimensional state of strain.

The final equation of motion for a layer subjected to harmonic stress in the coordinate system shown in Fig. 3 was derived by Sawicki (1987):

$$
\frac{d^{2} S}{D Z^{2}}=a \frac{S}{\sqrt{1-Z^{2}}}
$$

where

$$
a=\frac{\rho \omega^{2} H^{2}}{G_{0} \sqrt{\frac{1}{3}\left(1+2 K_{0}\right) \rho g H}},
$$

and $\rho$ is the bulk density, $\omega$ is the frequency of cyclic loading for harmonic oscillations, and $g$ is the gravitational acceleration.

The boundary conditions are as follows:

$$
\begin{aligned}
& S(Z=1)=0, \\
& S^{\prime}(Z=0)=-H \rho A_{0}=b .
\end{aligned}
$$

Solution of Eq. (8) with boundary condition (10) makes it possible to compute the distribution of the amplitude of the shear stress $S$ and subsequently, using formulae (2) and (5), the amplitude of the shear strain $\gamma_{0}$ within the soil stratum analyzed. Finally, applying formula (6), the relative change in porosity $\Phi$ and the settlement of the soil layer may be determined. The exact solution of this problem was given by Przewłócki and Knabe (1995), who used the Bessel functions. The final expressions for stress and strain amplitudes are:

$$
\begin{gathered}
S(Z)=\frac{b(1-Z) J_{2 / 3}\left[\left(4 \sqrt{\frac{a}{3}}\right)(1-Z)^{3 / 4}\right]}{\sqrt{a} J_{5 / 3}\left(4 \sqrt{\frac{a}{3}}\right)-J_{2 / 3}\left(4 \sqrt{\frac{a}{3}}\right)}, \\
\gamma_{0}(Z)=\frac{S(Z)}{G_{0} \sqrt{\frac{1}{3}\left(1+2 K_{0}\right) \rho_{0} g H(1-Z)}},
\end{gathered}
$$

where $J_{v}$ is a Bessel function that may be presented in the form of a series:

$$
J_{v}(y)=\left(\frac{y}{2}\right)^{v} \sum_{k=0}^{\infty} \frac{(-1)^{k}}{k ! \Gamma(v+k+1)}\left(\frac{y}{2}\right)^{2 k} .
$$


The settlement of a sublayer of thickness $t_{2}-t_{1}$ (see Fig. 3) can be easily obtained by integrating the porosity changes:

$$
s=\frac{n_{0}}{1-n_{0}} H \int_{Z_{1}}^{Z_{2}} \Phi d Z .
$$

For computing the settlement of a whole layer of thickness $H$, the limits of integration are assumed as $Z_{1}=0$ and $Z_{2}=1$. The solution is obtained numerically. If the layer of thickness $H$ is divided into $k$ strips of thickness $h_{i}$ and $\Phi_{i}$ is calculated in the middle of each strip, the total settlement is simply the sum of the settlements of individual strips:

$$
s=n_{0} \sum_{i=1}^{k} \Phi_{i} h_{i} .
$$

\section{Probabilistic Analysis of Soil Settlement}

The probabilistic analysis of the Izmit Bay soil settlement was performed basically by the PEM. According to this method, a continuous probability density function is replaced by a discrete function having the same first three central moments i.e. mean value, variance and skewness. The method applies appropriate weights to all evaluation points. It can also be implemented in response functions that are not explicit.

Rosenblueth (1975) proposed a PEM which concentrates the probability density function of a continuous random variable into two estimate points. He considered only correlated random variables whose skewness coefficients are zero. In the case of a function of $k$ random variables:

$$
y=g\left(x_{1}, x_{2}, \ldots, x_{k}\right)
$$

for each random variable $x_{i}$ there are two evaluation points denoted by $x_{i+}=m_{x_{i}}+\sigma_{x_{i}}$ and $x_{i-}=m_{x_{i}}+\sigma_{x_{i}}$, where $m_{x}$ is the mean value, and $\sigma_{x}$ is the standard deviation.

Function (16) should be applied to all possible combinations of evaluation points, i.e. $2^{k}$. The expected value and the variance of this function are expressed by the following formulae:

$$
m_{y} \approx \sum_{j=1}^{2^{k}} P^{j} y_{j}, \quad \sigma_{y}^{2} \approx \sum_{j=1}^{2^{k}} P^{j} y_{j}^{2}-m_{y}^{2},
$$

where:

$$
\begin{gathered}
P_{\left(s_{1} s_{2} \ldots x_{n}\right)}=\frac{1}{2^{n}}\left[1+\sum_{i=1}^{k-1} \sum_{j=i+1}^{k}\left(s_{i}\right)\left(s_{j}\right) r_{x_{i} x_{j}}\right], \\
s_{i}=\left\{\begin{array}{lll}
-1 & \text { for } \quad x_{i_{-}}=m_{x_{i}}-\sigma_{x_{i}} \\
+1 & \text { for } \quad x_{i_{+}}=m_{x_{i}}+\sigma_{x_{i}},
\end{array}\right.
\end{gathered}
$$


$r_{x_{i}, x_{j}}$ - cross-correlation coefficient between the random variables $X_{i}$ and $X_{j}$.

The above group of equations forms a basis for probabilistic analysis of settlement.

For the layer under consideration (Fig. 3), the following loading parameters required for calculations were specified: $H=10 \mathrm{~m}, g=9.81 \mathrm{~m} / \mathrm{s}^{2}, T=0.5 \mathrm{~s}, K_{0}=$ $0.344, A_{0}=0.2 \mathrm{~g}, N=100$. The soil model parameters $D_{1}$ and $D_{2}$, as well as $G_{0}$ and $n_{0}$, their mean values, standard deviations and all assumed correlation coefficients are given in section 3 .

The variation of the acceleration amplitude $A_{0}$ was assumed to be $v_{A_{0}}=0.1$. The corresponding mean values and the standard deviations are $m_{A_{0}}=1.962 \mathrm{~m} / \mathrm{s}^{2}$ and $\sigma_{A_{0}}=0.1962 \mathrm{~m} / \mathrm{s}^{2}$. Using the expressions presented in section 4 and the data described above, the deterministic value of the soil settlement was calculated: $s=$ $0.038 \mathrm{~m}$.

Two sets of probabilistic computations were performed.

First of all, due to a strong, almost full negative correlation between the random variables $D_{1}$ and $D_{2}$, one of them, the material coefficient $D_{2}$, was considered a function of $D_{1}$. The other variables of the problem were assumed uncorrelated. Thus the initial number of five random variables is reduced to four, i.e. $D_{1}, G_{0}, n_{0}, A_{0}$. Using PEM calculations, only 16 samples were considered. The mean value and the standard deviation obtained were $m_{s}=0.04327 \mathrm{~m}$ and $\sigma_{s}=0.00888 \mathrm{~m}$.

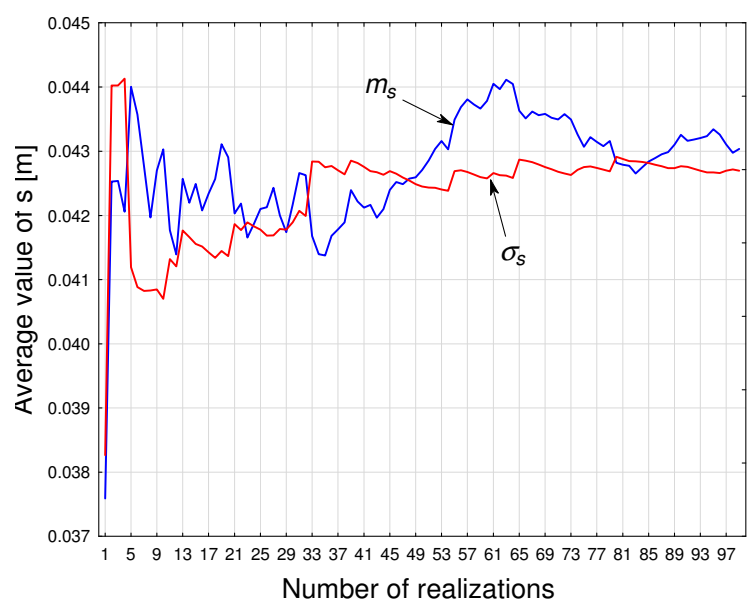

Fig. 4. Convergence analysis of the mean value and standard deviation of the soil settlement

The MCM was applied to verify the results. A total number of 100 sets of four random variables $D_{1}, G_{0}, n_{0}$, and $A_{0}$, were generated. In all cases, normal probability distributions were applied. The variable $D_{2}$ was assumed fully correlated with $D_{1}$. Soil settlements were calculated for 100 random sets. The result of convergence is presented in Figure 4. Based on the MCS, the mean value and standard deviation of the settlement were estimated as $0.04304 \mathrm{~m}$ and $0.00997 \mathrm{~m}$, respectively. The mean 
values obtained by the MCM and PEM are almost identical, but the standard deviations are diverse. It should be pointed out that the discrepancies result from the different definitions of correlation between the random variables $D_{1}$ and $D_{2}$.

Next, a set of calculations for different numbers of random variables were carried out. In all cases, the correlations between those parameters, described in section 3, were applied. The results presented in Table 1 prove that the standard deviation of the settlement grows with the increasing number of random parameters. As might be expected, the mean value of the settlement is higher than its deterministic equivalent.

Table 1. Results of the Rosenblueth PEM analysis for different numbers of random variables

\begin{tabular}{|c|c|c|c|c|}
\hline No. & $\begin{array}{c}\text { Random } \\
\text { variables }\end{array}$ & $n$ & $\begin{array}{c}\text { Average value of } \\
\text { settlement } m_{s}[\mathrm{~m}]\end{array}$ & $\begin{array}{c}\text { Standard deviation } \\
\text { of settlement } \sigma_{s}[\mathrm{~m}]\end{array}$ \\
\hline 1 & $D_{1}, D_{2}$ & 2 & 0.0439 & 0.0059 \\
\hline 2 & $D_{1}, D_{2}, G_{0}$ & 3 & 0.0443 & 0.0067 \\
\hline 3 & $D_{1}, D_{2}, G_{0}, n_{0}$ & 4 & 0.0444 & 0.0073 \\
\hline 4 & $D_{1}, D_{2}, G_{0}, n_{0}, A_{0}$ & 5 & 0.0443 & 0.0092 \\
\hline
\end{tabular}

In order to assess the correlation impact on the statistical parameters of the settlement, some additional computations were made, and their results are presented in Figs. 5 and 6 . The highest impact on both random settlement parameters is noted for the correlation between $D_{1}$ and $G_{0}$. Both values become weaker as the correlation between $D_{1}$ and $G_{0}$ and between $D_{1}$ and $n_{0}$ increases. These values grow up for higher correlation between $G_{0}$ and $n_{0}$.

a)

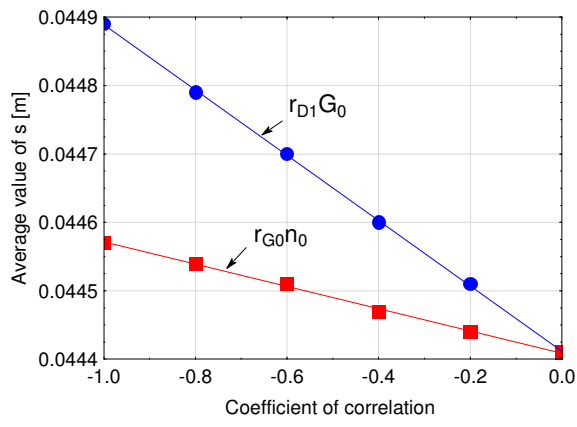

b)

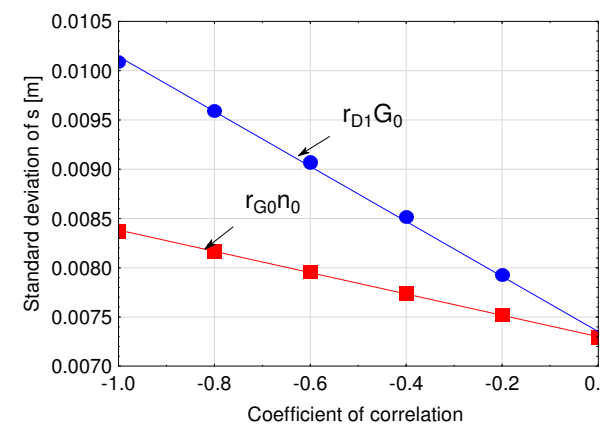

Fig. 5. Impact of correlation between coefficients $D_{1}$ and $G_{0}$, and between coefficient $D_{1}$ and porosity $n_{0}$ on a) mean value b) standard deviation

Further analysis was made to verify the influence of coefficient variations $v_{A_{0}}$ on both the mean value and the standard deviation of the settlement. The soil was described using four random variables. Calculations were performed for the correlations assumed in chapter 3. The results are presented in Fig. 7. It can be seen that, while the mean value of the settlement decreases, its standard deviation increases. 
a)

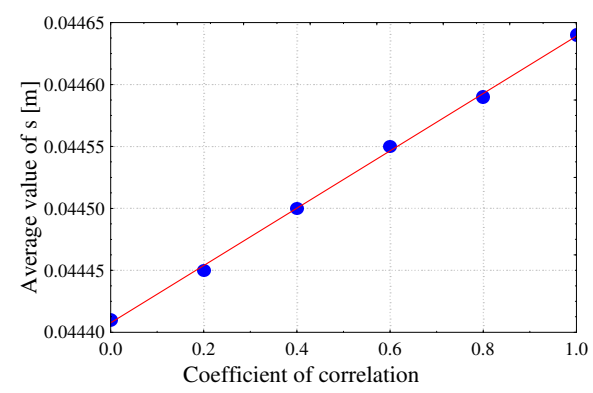

b)

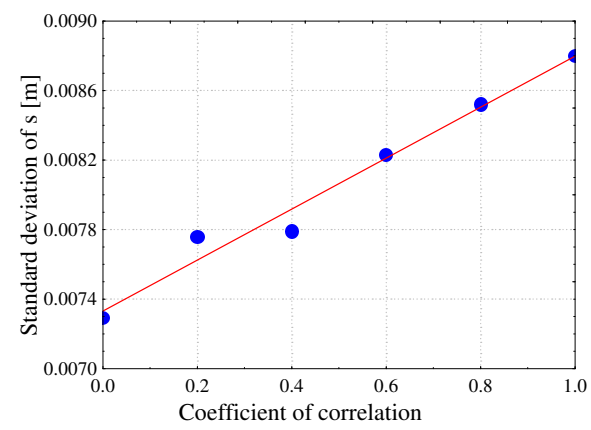

Fig. 6. Impact of correlation between coefficients $G_{0}$ and porosity $n_{0}$ on a) mean value b) standard deviation

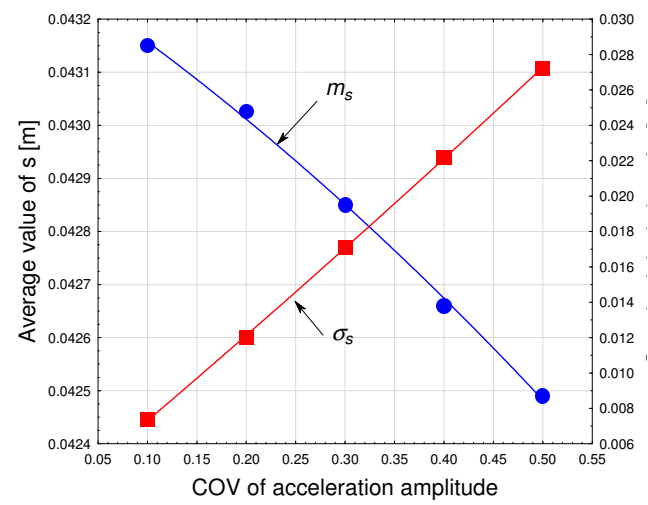

Fig. 7. Impact of the coefficient of variation of acceleration amplitude $v_{A_{0}}$ on the mean value and the standard deviation

Based on the results, a simplified version of the calculation procedure can be proposed. It is easy to notice that the variables with significant influence on the statistical characteristics of the settlement are the parameters related to the compaction model and the seismic load. Thus, only these two parameters, i.e. the material constant $D_{1}$ and the acceleration amplitude $A_{0}$, may be the ones considered as random. In such a case, there are only four samples analyzed. This causes a substantial reduction in computational effort. The mean value and standard deviation obtained are $m_{s}=0.04299 \mathrm{~m}$ and $\sigma_{s}=0.00711 \mathrm{~m}$, respectively. These results can be compared with those presented in Table 1 . Thus it can be stated that the simplified calculation results in the estimation of the settlement.

\section{Conclusions}

The PEM has proved to be an efficient tool for probabilistic geotechnical engineering applications. In contrast to the MCM and other standard simulation methods, only a small number of deterministic realizations are required here. The PEM is also an 
essential tool for implementing correlation between random variables. In this specific case, settlement calculations require tedious work, so the number of samples determines the efficiency of the method applied. Moreover, as the settlement is not given explicitly by closed-form analytical formulae, the problem cannot be solved by first-order second-moment techniques or similar methods. The PEM seems to be an appropriate tool for the analysis considered.

The statistical analysis proved a strong negative correlation between the material parameters $D_{1}$ and $D_{2}$. Their computed correlation coefficient $r_{D_{1} D_{2}}=-0.962$ denotes a practically full correlation - a deterministic relation between these two parameters. Random analysis may therefore be reduced to four parameters from the initial five.

Computations show a slight impact of subsoil parameters on the mean value of settlement. The mean value decreases while the coefficient of variation of acceleration amplitude increases. A slight influence of both porosity and the coefficient $G_{0}$ on the standard deviation of the settlement of the layer is also detected. This standard deviation is sensitive to random variation of the model parameters $D_{1}$ and $D_{2}$ and acceleration amplitude $A_{0}$. The latter relation is the most notable: the standard deviation of settlement increases proportionally to the coefficient of variation of $A_{0}$. The above conclusions and the direct relation between material parameters lead to a two-variable problem, without losing the accuracy of estimation. The statistical parameters of settlement are affected by correlation between distinct random variables. A vast parametric analysis presented in this paper shows that the standard deviation of settlement is significantly affected by the correlation between material parameters and the acceleration amplitude.

\section{References}

Baecher G. B., Christian J. T. (2003) Reliability and statistics in geotechnical engineering, Chichester: John Wiley \& Sons.

Bauer J., Puła W. (2000) Reliability with respect to settlement limit-states of shallow foundations on linearly-deformable subsoil, Computers and Geotechnics, 26 (3-4), 281-308.

Bourdeau P. L., Harr M. E. (1989) Stochastic theory of settlement of loose cohesionless soils, Géotechnique, 39 (4) 641-654.

Brząkała W., Puła W. (1996) A probabilistic analysis of foundation settlements, Computers and Geotechnics, 18 (4), 291-309.

Christian J. T., Baecher G. B. (1999) Point-estimate method as numerical quadrature, Journal of Geotechnical and Geoenvironmental Engineering, 125 (9), 779-786.

Fattah M.. (2010) Reliability-based design procedure of axially loaded piles, Journal of Engineering, 16 (1), 4462-4477.

Gibson W. (2011) Probabilistic methods for slope analysis and design, Australian Geomechanics, 46 (3), 1-11.

Gordon A., Fenton M., Griffiths D. V. (2002) Probabilistic Foundation Settlement on Spatially Random Soil, Journal of Geotechnical and Geoenvironmental Engineering, 128 (5), 381-390. 
Harr M. E. (1989) Probabilistic estimates for multivariate analyses, Appl. Math. Modelling, 13 (5), $313-318$.

Hong H. P. (1998) An efficient point estimate method for probabilistic analysis, Reliability Engineering and System Safety, 59 (3), 261-267.

Li K. S. (1992) Point Estimate Method for Calculating Statistical Moments, Journal of Engineering Mechanics, 118 (7), 506-1511.

Lind N. C. (1983) Modelling uncertainty in discrete dynamical systems, Appl. Math. Modelling, 7 (3), 146-152.

Martin G. R., Finn W. D. L., Seed H. B. (1975) Fundamentals of liquefuction under cyclic loading, Proc. ASCE, J. Geotech. Engng. Div., 101 (GT5), 423-428.

Przewłócki J. (1999) Reduction of dimension in random, elastic soil medium, International Journal of Solids and Structures, 36 (34), 5233-5254.

Przewłócki J. 2006 Problemy stochastycznej mechaniki gruntów - ocena niezawodności, Dolnośląskie Wydawnictwo Edukacyjne, Wrocław (in Polish).

Przewłócki J. Górski J. (1999) Stochastic FEM analysis of strip foundation, TASK Quarterly, 3 (2), 171-186.

Przewłócki J., Knabe W. (1995) Settlement of a soil stratum subjected to an earthquake, Int. Jnl. for Numerical and Analytical Methods in Geomechanics, 19, 813-821.

Rosenblueth E. (1975) Point estimates for probability moments, Proc. Nat. Acad. of Sci, 3812-3814.

Sawicki A. (1987) An egnineering model for compaction of sand under cyclic loading, Engineering Transactions, 35 (94), 677-693.

Sawicki A., Morland W. (1986) Dynamic shear response of a granular column, Proc. US Eng. Found. Conf., Henniker. NH.

Sawicki A., Świdziński W. (2006) A study on liquefaction susceptibility of some soils from the coast of Marmara sea, Bulletin of the Polish Academy of Sciences, 54 (4), 405-418.

Sawicki A., Świdziński W. (2007) A simple mathematical model for assessment of seismic-induced liquefaction of soils, ASCE J Waterway, Port, Coastal and Ocean Engineering, LIMAS Special Issue, 133 (1), 50-54.

Sayed S., Dodagoudar G. R., Rajagopal K. (2008) Reliability analysis of reinforced soil walls under static and seismic forces, Geosynthetics International, 15 (4), 246-257.

Suchomel R., Mašín (2011) Probabilistic analyses of a strip footing on horizontally stratified sandy deposit using advanced constitutive model, Computers and Geotechnics, 38 (3), 363-374.

Wang J. P., Huang D. (2012) RosenPoint: A Microsoft Excel-based program for the Rosenblueth point estimate method and an application in slope stability analysis, Computers and Geosciences, 48, 239-243. 